\title{
Myocardial perfusion MRI using SW-CG-HYPR for the detection of coronary artery disease
}

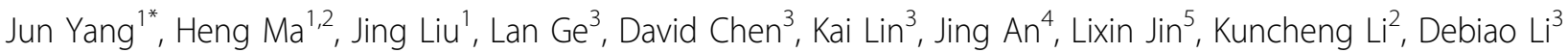 \\ From 15th Annual SCMR Scientific Sessions \\ Orlando, FL, USA. 2-5 February 2012
}

\section{Summary}

In this work, we have prospectively examined the diagnostic value of adenosine-induced stress myocardial perfusion MRI using SW-CG-HYPR in 50 patients with suspected CAD. Using the high-resolution, whole left ventricle SW-CG-HYPR method, perfusion MRI was able to depict hemodynamically relevant coronary artery stenosis with an accuracy of $90 \%$ and $93 \%$ based on perpatient and per-vessel analyses, respectively, using $x$-ray coronary angiography as the reference standard.

\section{Background}

Myocardial perfusion MRI with SW-CG-HYPR allows increased spatial coverage (whole left ventricular coverage), improved temporal and spatial resolution and signal-to-noise ratio, and reduced motion artifacts. The accuracy of this technique for detecting coronary artery disease (CAD) has not been determined in a large number of patients. The purpose of this study was to prospectively evaluate the diagnostic performance of myocardial perfusion MRI with SW-CG-HYPR in patients with suspected CAD.

\section{Methods}

Fifty consecutive patients ( 28 men and 22 women; mean age, $56 \pm 16$ years) who were scheduled for coronary angiography with suspected CAD underwent myocardial perfusion MRI with SW-CG-HYPR at 3.0T. Perfusion defects were interpreted qualitatively by 2 blinded observers and were correlated to $\mathrm{x}$-ray angiographic stenoses $\geq 50 \%$.

\section{Results}

The prevalence of CAD was $56 \%$. In the per-patient analysis, the sensitivity, specificity, positive predictive value, negative predictive value, and accuracy of SWCG-HYPR myocardial perfusion imaging were 96\% (95\% confidence interval $[\mathrm{CI}] 82 \%$ to $100 \%), 82 \%$ (95\% CI $60 \%$ to $95 \%$ ), $87 \%$ (95\% CI $70 \%$ to 96 ), $95 \%$ (95\% CI $74 \%$ to $100 \%$ ), and $90 \%$ (95\% CI $82 \%$ to $98 \%$ ), respectively. In the per-vessel analysis, these values were $98 \%$ (95\% CI $91 \%$ to $100 \%$ ), $89 \%$ (95\% CI $80 \%$ to $94 \%$ ), $86 \%$ ( $95 \%$ CI $76 \%$ to $93 \%$ ), $99 \%$ ( $95 \%$ CI $93 \%$ to $100 \%$ ), and 93\% (95\% CI $89 \%$ to $97 \%$ ), respectively.

\section{Conclusions}

Myocardial perfusion MRI using SW-CG-HYPR allows whole left ventricular coverage and high resolution, and has high diagnostic accuracy in patients with suspected CAD.

\section{Funding}

This work was supported by National Institute of Health (NIBIB EB002623) and National Natural Science Foundation of China (30828009).

\section{Author details \\ ${ }^{1}$ Yuhuangding Hospital, Yantai, China. ${ }^{2}$ Capital Medical University, Beijing, China. ${ }^{3}$ University of California, Los Angeles, CA, USA. ${ }^{4}$ Siemens Mindit Magnetic Resonance, Shenzhen, China. ${ }^{5}$ Siemens Limited China, Shanghai, China.}

Published: 1 February 2012

doi:10.1186/1532-429X-14-S1-T5

Cite this article as: Yang et al:: Myocardial perfusion MRI using SW-CGHYPR for the detection of coronary artery disease. Journal of

Cardiovascular Magnetic Resonance 2012 14(Suppl 1):T5.

${ }^{1}$ Yuhuangding Hospital, Yantai, China

Full list of author information is available at the end of the article

(C) 2012 Yang et al; licensee BioMed Central Ltd. This is an open access article distributed under the terms of the Creative Commons Attribution License (http://creativecommons.org/licenses/by/2.0), which permits unrestricted use, distribution, and reproduction in any medium, provided the original work is properly cited. 